OPEN ACCESS

Edited by:

Signe Altmäe,

University of Granada, Spain

Reviewed by:

Qi Yu,

Peking Union Medical College

Hospital (CAMS), China

Alberto Sola-Leyva,

University of Granada, Spain

*Correspondence:

Xiaocan Lei

liulincun@163.com

Shun Zhang

artzhangshun@glmc.edu.cn

${ }^{\dagger}$ These authors have contributed equally to this work

Specialty section: This article was submitted to Reproduction,

a section of the journal

Frontiers in Physiology

Received: 17 January 2020 Accepted: 06 April 2020

Published: 20 May 2020

Citation:

Huo P, Zhu Y, Liang C, Yao J, Le J, Qin L, Lei X and Zhang S (2020) Non-invasive Amino Acid Profiling of Embryo Culture Medium Using HPLC Correlates With Embryo Implantation Potential in Women Undergoing in vitro Fertilization.

Front. Physiol. 11:405. doi: 10.3389/fphys.2020.00405

\section{Non-invasive Amino Acid Profiling of Embryo Culture Medium Using HPLC Correlates With Embryo Implantation Potential in Women Undergoing in vitro Fertilization}

\author{
Peng Huo't, Yunshan Zhu'2t, Chengqin Liang ${ }^{3}$, Jun $\mathrm{Yao}^{2}$, Jianghua Le², Linyuan Qin', \\ Xiaocan Lei ${ }^{4 *}$ and Shun Zhang ${ }^{2 *}$ \\ ${ }^{1}$ School of Public Health, Guilin Medical University, Guilin, China, ${ }^{2}$ Department of Reproductive Medical Center, \\ The Affiliated Hospital of Guilin Medical University, Guilin, China, ${ }^{3}$ College of Pharmacy, Guilin Medical University, Guilin, \\ China, ${ }^{4}$ Clinical Anatomy \& Reproductive Medicine Application Institute, Department of Histology and Embryology, University \\ of South China, Hengyang, China
}

This study aimed to determine the correlation between amino acid profiling of a 3-dayold embryo culture medium and embryo implantation potential in women undergoing in vitro fertilization (IVF). The data of 98 patients who received IVF treatment in our hospital from December 2015 to February 2017 were retrospectively analyzed. The 98 patients were grouped into a pregnant group (gemellary pregnancy), a non-pregnant group (non-pregnancy), and a blank control group. The amino acids from a 3-day-old embryo culture medium and blank control medium were collected and were analyzed using high performance liquid chromatography (HPLC). The HPLC results showed that amino acids including aspartate (ASP), serine (SER), glycine (GLY), histidine (HIS), taurine (TAU), arginine (ARG), threonine (THR), alanine (ALA), and proline (PRO) were detected in the 3-day-old embryo culture medium and blank control medium. There are significant differences between the pregnant group and non-pregnant group in peak height $(H)$ SER, surface area (S)-ASP, S-SER, S-HIS, and S-ALA. The discrimination analysis according to the peak height and peak area of amino acids revealed that the prediction rate of the pregnant group, non-pregnant group, and blank control group were 82.7, 95.7, and $100 \%$. Further, by using the principal component analysis, we found that the prediction rate in these three groups were $90.4,91.3$, and 100\%. Our data may suggest that using amino acid concentrations for principal component analysis and discriminant analysis has high accuracy in predicting the relationship between amino acid fingerprint and embryo implantation potential.

Keywords: amino acid, embryo development potential, in vitro fertilization, gemellary pregnancy, principal component analysis 


\section{INTRODUCTION}

In recent years, infertility has become a worldwide medical problem affecting about $10-15 \%$ of people globally (Scoccia, 2015), while the incidence of infertility among people of reproductive age in China is $12.5-15 \%$ (Zhou et al., 2018). With the continuous advancement of medical research and technology, assisted reproductive technology (ART) has become the primary method for the treatment of infertility (Liberman et al., 2017). In ART, multiple pregnancies are the major concern, and the pregnancy complications include preterm delivery, low birth weight, and an increased risk for cerebral palsy. Reducing the incidence of multiple gestations while improving the pregnancy rates is an important focus on infertility research. Current embryo assessment strategies are largely based on embryo morphology (Rodgaard et al., 2015). However, the assessment on embryo morphology cannot reliably predict the embryo implantation potential. Though embryo transfer with two morphologically good embryos can produce relatively satisfying pregnancy rates, this can lead to $20-30 \%$ gemellary pregnancy, which threatens the health of mothers and babies. Therefore, development of an objective, accurate, fast, and affordable test that can aid in the assessment of embryo developmental potential for single embryo transfer is a significant aim of reproductive medicine.

Metabolomics, genomics, and proteomics are important components of system biology. Metabolomics can detect changes of metabolic substances in living organisms, which can reflect the intrinsic or extrinsic interactions of organisms, organs, and cells. Metabolites are intermediates or end products produced by organisms under specific conditions (Egea et al., 2014). In reproductive studies, the metabolic process of embryos can be determined by analyzing their related metabolites, which may provide a non-invasive method for predicting embryonic developmental capacity (Nel-Themaat and Nagy, 2011; Li et al., 2018). In the field of assisted reproduction, the development of embryos can be evaluated by detecting glucose, fatty acids, and amino acids in an embryo culture (Armstrong et al., 2018; De Rose et al., 2018). At present, the detection of amino acids can help to evaluate the development of embryos to a certain extent and help to judge the pregnancy outcome. Boyama et al. (2016) examined the relationship between the concentration of homocysteine ( $\mathrm{Hcy}$ ) in embryonic media and pregnancy outcomes, indicating that the concentration of Hcy is inversely related to pregnancy outcome (Borowiecka et al., 2012). With the deepening of research, amino acid metabolism, as a reference indicator for embryo evaluation, has been increasingly used in clinical work (Sturmey et al., 2008). The measurement of amino acid turnover has been suggested as non-invasive way to assess embryo viability (Sturmey et al., 2008). However, to date, how the changes of amino acid profiles during human embryo development affect pregnancy outcomes in IVF has not yet been determined.

In this study, we recruited 98 patients who had received IVFembryo transfer (ET) treatment in our hospital and analyzed the concentrations of the amino acids in the embryo culturing medium by using high performance liquid chromatography (HPLC). By using principal component analysis (PCA), the relationship between amino acid fingerprint and embryo development potential was further explored. The present study may provide a relevant basis for the selection of high-quality embryos with high implantation potential during IVF by analyzing the amino acid fingerprint.

\section{MATERIALS AND METHODS}

\section{Study Subjects}

The present study recruited 98 women who were infertile and were receiving IVF-ET treatment for pregnancy between December 2015 and February 2017 in the Reproductive Center of the Affiliated Hospital of Guilin Medical University. This study was approved by Ethics Committee of the Affiliated Hospital of Guilin Medical University (No. GLMC20183040), and written informed consent was signed by each patient. Inclusion criteria were: (1) age between 25-35 years old, (2) body mass index between $18-29 \mathrm{~kg} / \mathrm{m}^{2}$, (3) primary infertile women with major indications for IVF, (4) women with fallopian tube problems and required IVF for pregnancy, (5) more than 5 oocytes were collected during IVF, and (6) the couples had no abnormalities in the chromosomes. Exclusion criteria were: (1) patients with a history of recurrent pregnancy loss; (2) patients with impaired ovulation; (3) patients with ovarian hyperstimulation syndrome; and (4) any significant systemic disease, endocrine, or metabolic disorder.

\section{Collection of Oocytes}

Ovarian stimulation was performed using recombinant follicle stimulating hormone (FSH) with highly purified urinary gonadotropin using a luteal long protocol with a gonadotropinreleasing hormone (GnRH) agonist. When two leading follicles reached a mean diameter of $\geq 18 \mathrm{~mm}$ or three leading follicles reached a mean diameter of $\geq 16 \mathrm{~mm}, 5000-10000$ IU of recombinant human chorionic gonadotropin (hCG) was injected. Ultrasonography-guided transvaginal oocyte retrieval was performed $36 \mathrm{~h}$ after hCG administration.

\section{Sperm Collection}

Semen was collected on the day of oocyte retrieval according to the standard protocols of IVF. Following liquefaction, the whole semen was mixed with the G-IVF plus solution (Vitrolife, Sweden). The sperm was allowed to swim-up for $40 \mathrm{~min}$ at $37^{\circ} \mathrm{C}$ in an incubator with $6 \% \mathrm{CO}_{2}$. The supernatant with swim-up sperms were collected and centrifuged at $200 \mathrm{~g}$ for $5 \mathrm{~min}$. The sperm pellet was re-suspended in $1.0 \mathrm{ml} \mathrm{G}$-IVF plus solution and the resuspended pellet was later used for IVF/intracytoplasmic sperm injection (ICSI).

\section{ET and Culture Medium Collection}

The fertilization was performed using IVF/ICSI, and the embryos were transferred 3 days after oocyte retrieval. Embryo quality was evaluated by morphological criteria based on the degree of fragmentation and the regularity of blastomeres on day three after fertilization. The embryos were graded as follows: grade A, $0 \%$ anucleate fragments, regularity of blastomeres, and no apparent morphological abnormality; grade B, $<20 \%$ 
anucleate fragments, regularity of blastomeres, and no apparent morphological abnormality. The embryos with grade A or grade $\mathrm{B}$ were included in the study. The culture medium for embryos at D3 after embryo transfer was collected. For the blank control group, the culture medium without embryos culturing were used in this study. All the collected medium was stored in liquid nitrogen before amino acids analysis.

\section{Follow-Up Study}

Clinical pregnancy was defined as the positive hCG test from urine at 14 day after ET and the presence of one or more gestational sacs at 28 day after ET. Miscarriage was defined as pregnancy loss before 12 weeks of gestation. In the study, a total of 52 patients who had a gemellary pregnancy were defined as being in the pregnant group, and the 46 patients with two embryos but who had lost their pregnancy before 12 weeks of gestation were defined as the non-pregnant group.

\section{Solvents, Reagents and HPLC Analysis}

All solvents used were of HPLC grade and were purchased from Sigma-Aldrich (St. Louis, United States). The 18 individual amino acid standards, including histidine (His), serine (Ser), arginine (Arg), glycine (Gly), aspartate (Asp), glutamate (Glu), threonine (The), alanine (Ala), proline (Pro), cystine (Cys), lysine (Lys), tyrosine (Tyr), methionine (Met), valine (Val), isoleucine (Ile), leucine (Leu), glutamine (Gln), and phenylalanine (Phe), were purchased from Sigma-Aldrich (St. Louis, United States). Sample preparation from culture media was performed using the Water AccQ-Tag ${ }^{\mathrm{TM}}$ Ultra Derivatisation Kit (Waters, Milford, United States). Firstly, the derivatization reagent (AccQTag Ultra Reagent power, AQC) was reconstituted in AccQTag Reagent Diluent (acetonitrile). Standard sample mixtures $(10 \mu \mathrm{L})$ or the culture media $(10 \mu \mathrm{L})$ from the samples was mixed with $20 \mu \mathrm{L}$ of derivatization and $70 \mu \mathrm{L}$ of AccQTag Ultra Borate Buffer in a tapered glass vial. The mixture was vortexed twice with a 1 min interval standing at room temperature and incubated in a heating block for $10 \mathrm{~min}$ at $55^{\circ} \mathrm{C}$. Chromatographic separation was achieved by injection of $1 \mu \mathrm{L}$ of sample or standard sample using an ACCQTAG ULTRA C18 $1 \mu \mathrm{m}, 2.1 \times 100 \mathrm{~mm}$ column at $37^{\circ} \mathrm{C}$ using a gradient mobile phase consisting of $100 \%$ HPLC-grade water (A), 100\% AccQ-Tag Ultra Eluent A (B), idle (C), and 100\% HPCL-grade acetonitrile (D). Gradient conditions are shown in Table 1. A Tunable UV detector was used to detect sample absorbance at $260 \mathrm{~nm}$ in single wavelength mode. Data acquisition and processing was performed using Waters Empower 2 chromatography software using the manufacturerdefined method for protein hydrolysates. Individual amino acid elution times were manually verified in standard samples prior to processing with the manufacturer defined methodology to quantitate amino acids. The peak height $(\mathrm{H})$ and surface area (S) for corresponding amino acids were extracted and was exported to Excel.

\section{Statistical Analysis}

All the data were analyzed using the SPSS 20.0 software (IBM, Armonk, United States). The data were presented as
TABLE 1 | Chromatographic gradient elution profile for amino acid analysis.

\begin{tabular}{lrrrrr}
\hline Time (min) & A (\%) & B (\%) & C (\%) & D (\%) & Flow (ml/min) \\
\hline 0.00 & 0.0 & 0.0 & 0.0 & 0.0 & 1.00 \\
11.00 & 0.0 & 100.0 & 0.0 & 0.0 & 1.00 \\
11.01 & 0.0 & 99.0 & 0.0 & 1.0 & 1.00 \\
21.00 & 0.0 & 95.0 & 0.0 & 5.0 & 1.00 \\
22.00 & 0.0 & 91.0 & 0.0 & 9.0 & 1.00 \\
28.50 & 0.0 & 83.0 & 0.0 & 17.0 & 1.00 \\
38.00 & 0.0 & 83.0 & 0.0 & 17.0 & 1.00 \\
38.01 & 40.0 & 0.0 & 0.0 & 60.0 & 1.00 \\
41.00 & 40.0 & 0.0 & 0.0 & 60.0 & 1.00 \\
41.01 & 0.0 & 100.0 & 0.0 & 0.0 & 1.00 \\
68.00 & 0.0 & 100.0 & 0.0 & 0.0 & 1.00 \\
68.01 & 40.0 & 0.0 & 0.0 & 60.0 & 1.00 \\
100.00 & 40.0 & 0.0 & 0.0 & 60.0 & 1.00 \\
\hline
\end{tabular}

mean \pm standard deviation. Significant differences between two groups were analyzed using an unpaired $t$-test. $P<0.05$ was considered statistically significant.

\section{RESULTS}

\section{General Characteristics of the Recruited Patients}

In the present study, 98 patients were recruited in our analysis, and 52 patients were classified into the pregnant group and 46 patients were classified into the non-pregnant group. The general characteristics of all the patients were shown in Table 2 and there was no significant difference in these variables between the pregnant group and non-pregnant group (Table 2).

\section{Construction of Standard Curve for Determination of Amino Acid Concentrations}

The concentrations of $0.1,0.2,0.3,0.4$, and $0.45 \mu \mathrm{mol} / \mathrm{ml}$ for amino acid standards were used to construct the standard curve. The construct standard curves were shown in Table 3.

\section{Detection of Amino Acids in the Culture Medium}

In the present study, eight amino acids including SER, GLY, HIS, TAU, ARG, THR, ALA, and PRO were detected in the culture medium from the blank control group, non-pregnant group, and pregnant group. The peak height and surface area of the HPLC results for amino acids were all determined (Table 4).

\section{Discriminant Analysis Using Amino Acid Data}

First of all, we included the values for peak height and surface area of the detected amino acids for the discriminant analysis. The results of the discriminant analysis were shown in Figure $\mathbf{1 .}$ The cross-validation results showed that the prediction rate for the blank control group, non-pregnant group, and pregnant 
TABLE 2 | Clinical parameters of the recruited subjects.

\begin{tabular}{|c|c|c|c|}
\hline Variables & $\begin{array}{l}\text { Gemellary } \\
\text { pregnancy }\end{array}$ & $\begin{array}{l}\text { Non- } \\
\text { pregnancy }\end{array}$ & $P$ values \\
\hline Age (years) & $29.53 \pm 4.52$ & $30.04 \pm 4.56$ & 0.609 \\
\hline Duration of infertility (years) & $3.1 \pm 1.97$ & $3.17 \pm 2.20$ & 0.874 \\
\hline Body mass index $\left(\mathrm{kg} / \mathrm{m}^{2}\right)$ & $21.84 \pm 3.00$ & $21.90 \pm 2.92$ & 0.916 \\
\hline Baseline FSH levels (IU/L) & $6.22 \pm 1.25$ & $5.96 \pm 1.47$ & 0.404 \\
\hline Baseline LH levels (IU/L) & $5.48 \pm 2.03$ & $4.71 \pm 1.88$ & 0.071 \\
\hline Baseline E2 levels (pg/mL) & $141.52 \pm 47.48$ & $132.42 \pm 57.27$ & 0.447 \\
\hline Initial dose of gonadotropin (IU) & $194.17 \pm 59.25$ & $186.59 \pm 54.92$ & 0.540 \\
\hline $\begin{array}{l}\text { FSH levels on the day of } \\
\text { starting gonadotropin } \\
\text { administration (IU/L) }\end{array}$ & $3.46 \pm 1.033$ & $3.345 \pm 1.41$ & 0.709 \\
\hline $\begin{array}{l}\text { LH levels on the day of starting } \\
\text { gonadotropin administration } \\
\text { (IU/L) }\end{array}$ & $1.57 \pm 0.81$ & $1.62 \pm 0.70$ & 0.730 \\
\hline $\begin{array}{l}\text { E2 levels on the day of starting } \\
\text { gonadotropin administration } \\
(\mathrm{pg} / \mathrm{ml})\end{array}$ & $54.11 \pm 35.96$ & $50.31 \pm 35.69$ & 0.628 \\
\hline $\begin{array}{l}\text { Progesterone levels on the day } \\
\text { of starting gonadotropin } \\
\text { administration (IU/L) }\end{array}$ & $1.70 \pm 0.76$ & $1.64 \pm 0.61$ & 0.682 \\
\hline $\begin{array}{l}\text { LH levels on the day of HCG } \\
\text { administration (IU/L) }\end{array}$ & $2.28 \pm 1.86$ & $1.85 \pm 1.02$ & 0.140 \\
\hline $\begin{array}{l}\text { E2 levels on the day of HCG } \\
\text { administration }(\mathrm{ng} / \mathrm{mL})\end{array}$ & $15.18 \pm 7.93$ & $17.13 \pm 7.49$ & 0.248 \\
\hline $\begin{array}{l}\text { Progesterone levels on the day } \\
\text { of } \mathrm{HCG} \text { administration (IU/L) }\end{array}$ & $3.03 \pm 0.89$ & $3.02 \pm 0.89$ & 0.971 \\
\hline $\begin{array}{l}\text { Endometrial thickness on the } \\
\text { day of embryo implantation }\end{array}$ & $10.26 \pm 2.02$ & $10.70 \pm 2.38$ & 0.374 \\
\hline
\end{tabular}

group were $100,95.7$, and $80.8 \%$, respectively. In addition, the overall prediction rate is $95.9 \%$, and the accuracy of the crossvalidation is $91.8 \%$.
TABLE 4 | Concentrations of amino acids in the recruited subjects.

\begin{tabular}{lccc}
\hline & Pregnant & Non-pregnant & P values \\
\hline S-ASP & $107.71 \pm 36.12$ & $126.31 \pm 45.58$ & 0.028 \\
S-SER & $124.16 \pm 43.27$ & $42.56 \pm 24.52$ & 0 \\
S-GLY & $88.46 \pm 60.34$ & $86.35 \pm 50.44$ & 0.853 \\
S-HIS & $130.23 \pm 37.54$ & $80.74 \pm 100.57$ & 0.001 \\
S-TAU & $37.63 \pm 15.63$ & $34.72 \pm 10.30$ & 0.287 \\
S-ARG & $46.49 \pm 17.64$ & $41.15 \pm 14.91$ & 0.112 \\
S-THR & $82.64 \pm 34.62$ & $85.78 \pm 27.57$ & 0.625 \\
S-ALA & $118.12 \pm 63.09$ & $153.90 \pm 42.21$ & 0.002 \\
S-PRO & $41.06 \pm 20.10$ & $47.74 \pm 18.75$ & 0.095 \\
H-ASP & $6.28 \pm 2.06$ & $5.79 \pm 2.65$ & 0.315 \\
H-SER & $8.99 \pm 2.83$ & $3.63 \pm 2.37$ & 0 \\
H-GLY & $7.01 \pm 2.13$ & $6.27 \pm 3.99$ & 0.256 \\
H-HIS & $5.14 \pm 3.04$ & $4.99 \pm 3.10$ & 0.812 \\
H-TAU & $3.02 \pm 1.43$ & $2.94 \pm 1.07$ & 0.767 \\
H-ARG & $3.96 \pm 1.48$ & $3.88 \pm 1.69$ & 0.805 \\
H-THR & $8.80 \pm 4.10$ & $8.69 \pm 3.01$ & 0.884 \\
H-ALA & $11.47 \pm 5.69$ & $11.73 \pm 3.11$ & 0.783 \\
H-PRO & $3.78 \pm 1.50$ & $3.59 \pm 1.12$ & 0.47 \\
\hline
\end{tabular}

In terms of the peak height and surface area, the values of S-SER, S-HIS, and H-SER were significantly higher in the pregnant group than that in the non-pregnant group, while the values of S-ASP and S-ALA were significantly lower in the pregnant group than that in the non-pregnant group (Table 4). In this regard, we included S-ASP, S-SER, S-HIS, S-ALA, and H-SER to perform the discriminant analysis. The results of the discriminant analysis were shown in Figure 2. The cross-validation results showed that the prediction rates for the blank control group, non-pregnant group, and pregnant group were 100, 95.7, and $82.7 \%$, respectively. The overall

TABLE 3 | Standard curve construction for amino acids.

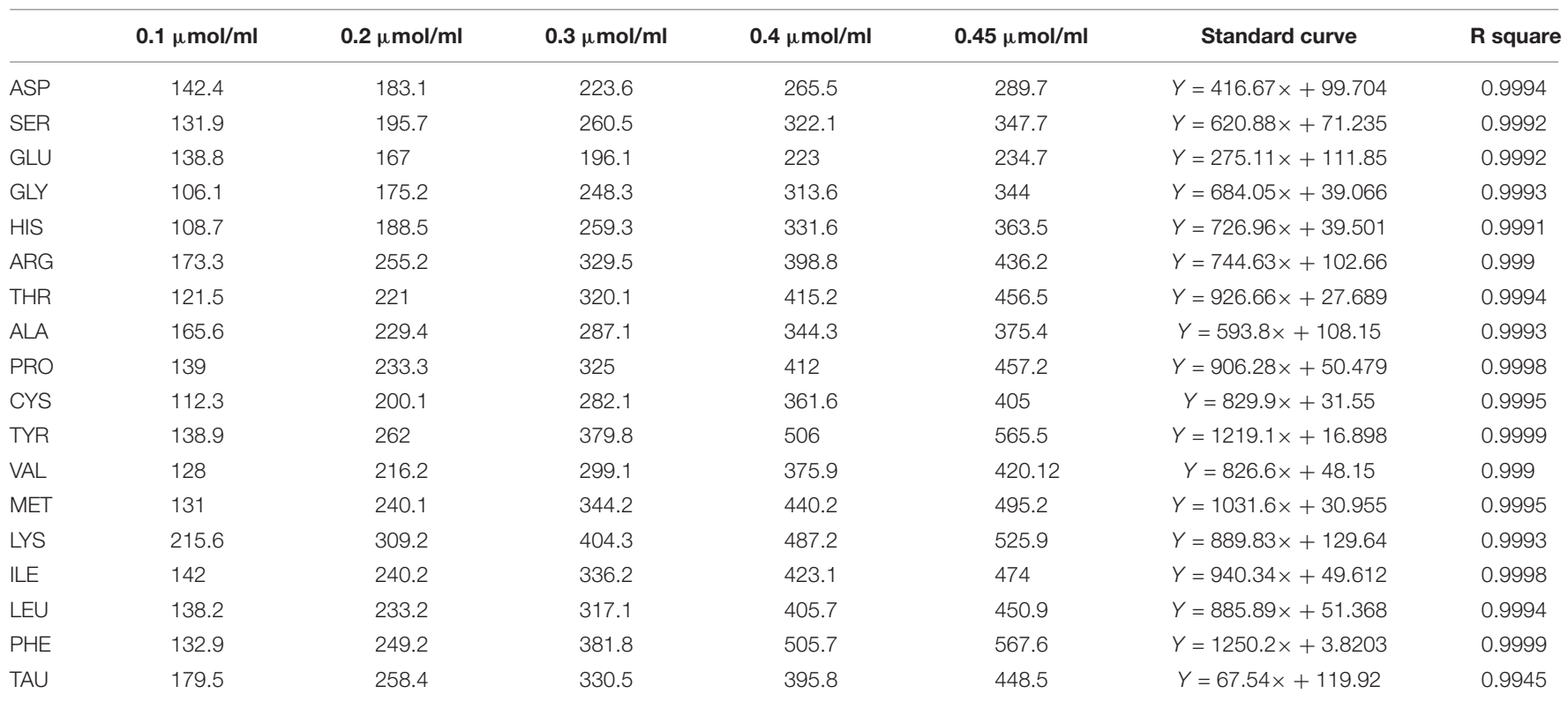




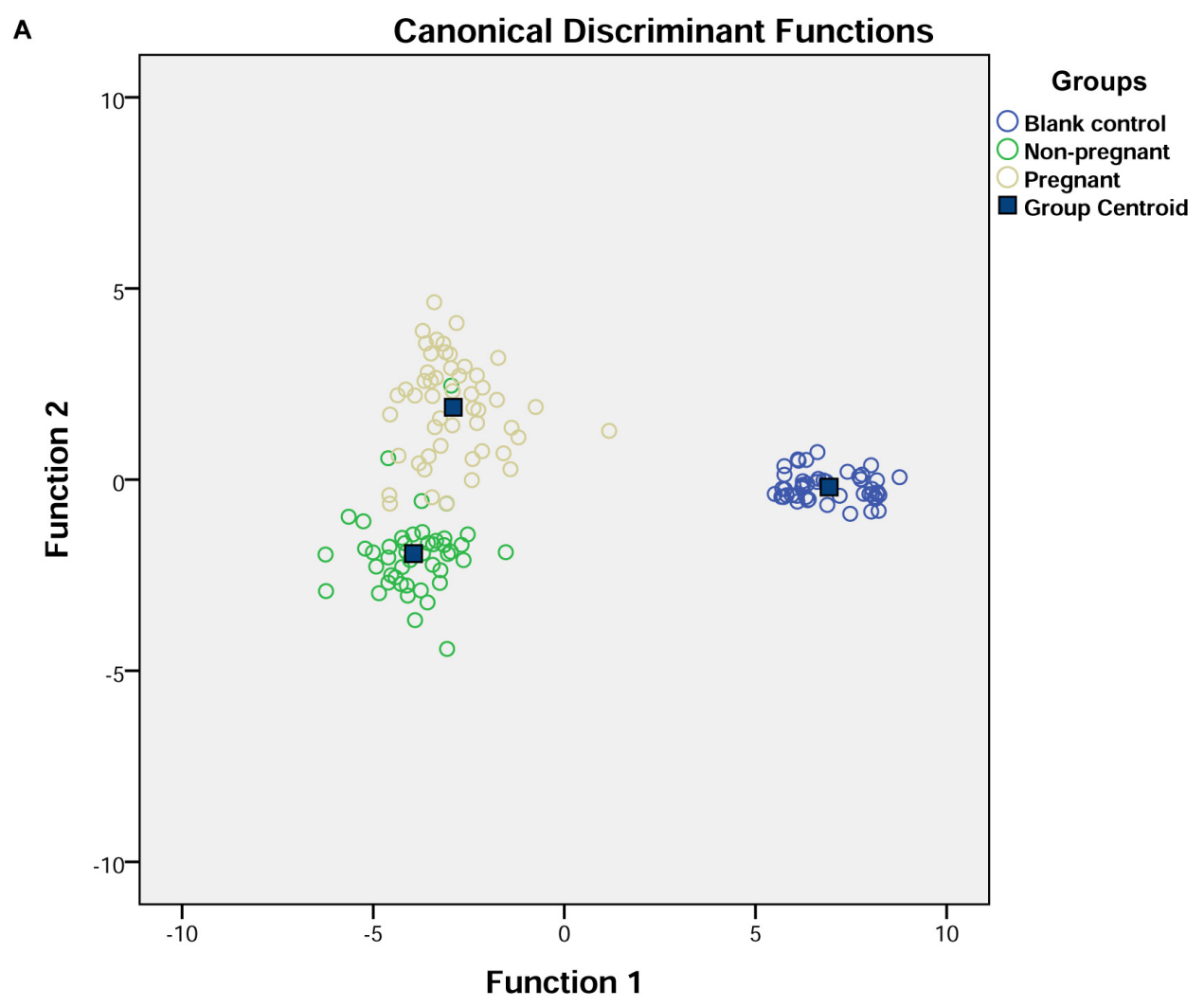

\begin{tabular}{|c|c|c|c|c|c|c|}
\hline \multirow[t]{3}{*}{ B } & \multicolumn{6}{|c|}{ Classification Results $^{a, c}$} \\
\hline & & \multirow[b]{2}{*}{ Groups } & \multicolumn{3}{|c|}{ Predicted Group Membership } & \multirow[b]{2}{*}{ Total } \\
\hline & & & Blank control & Non-pregnant & Pregnant & \\
\hline \multirow[t]{6}{*}{ Original } & \multirow[t]{3}{*}{ Count } & Blank control & 48 & 0 & 0 & 48 \\
\hline & & Non-pregnant & 0 & 44 & 2 & 46 \\
\hline & & Pregnant & 0 & 4 & 48 & 52 \\
\hline & \multirow[t]{3}{*}{$\%$} & Blank control & 100.0 & .0 & .0 & 100.0 \\
\hline & & Non-pregnant & .0 & 95.7 & 4.3 & 100.0 \\
\hline & & Pregnant & .0 & 7.7 & 92.3 & 100.0 \\
\hline \multirow[t]{6}{*}{ Cross-validated $^{\mathrm{b}}$} & \multirow[t]{3}{*}{ Count } & Blank control & 48 & 0 & 0 & 48 \\
\hline & & Non-pregnant & 0 & 44 & 2 & 46 \\
\hline & & Pregnant & 1 & 9 & 42 & 52 \\
\hline & \multirow[t]{3}{*}{$\%$} & Blank control & 100.0 & .0 & .0 & 100.0 \\
\hline & & Non-pregnant & .0 & 95.7 & 4.3 & 100.0 \\
\hline & & Pregnant & 1.9 & 17.3 & 80.8 & 100.0 \\
\hline
\end{tabular}

a. $95.9 \%$ of original grouped cases correctly classified.

b. Cross validation is done only for those cases in the analysis. In cross validation, each case is classified by the functions derived from all cases other than that case.

c. $91.8 \%$ of cross-validated grouped cases correctly classified.

FIGURE 1 | Discriminant analysis of amino acid profiles in the culture medium. (A) Canonical discriminant functions. (B) Classification results. 


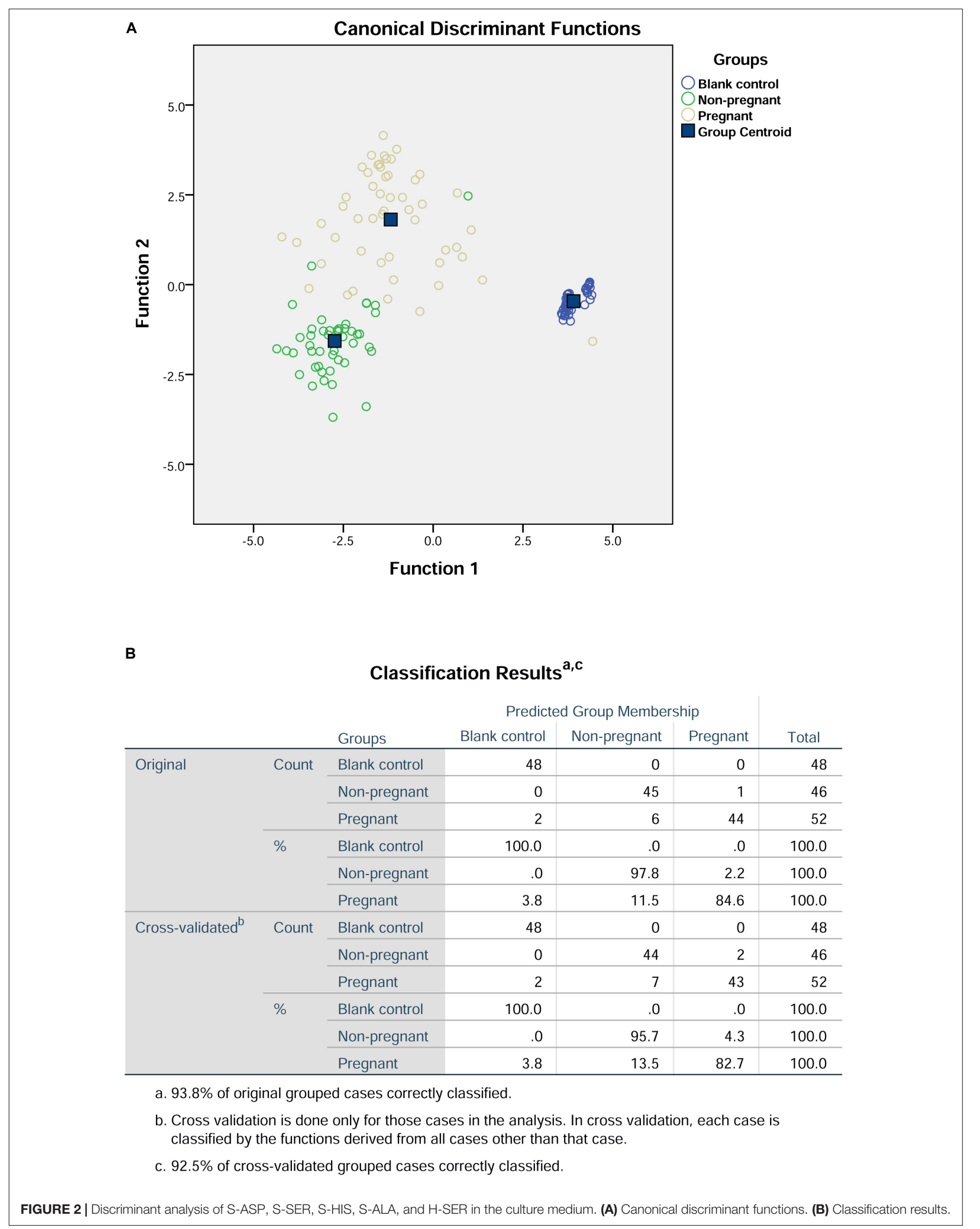




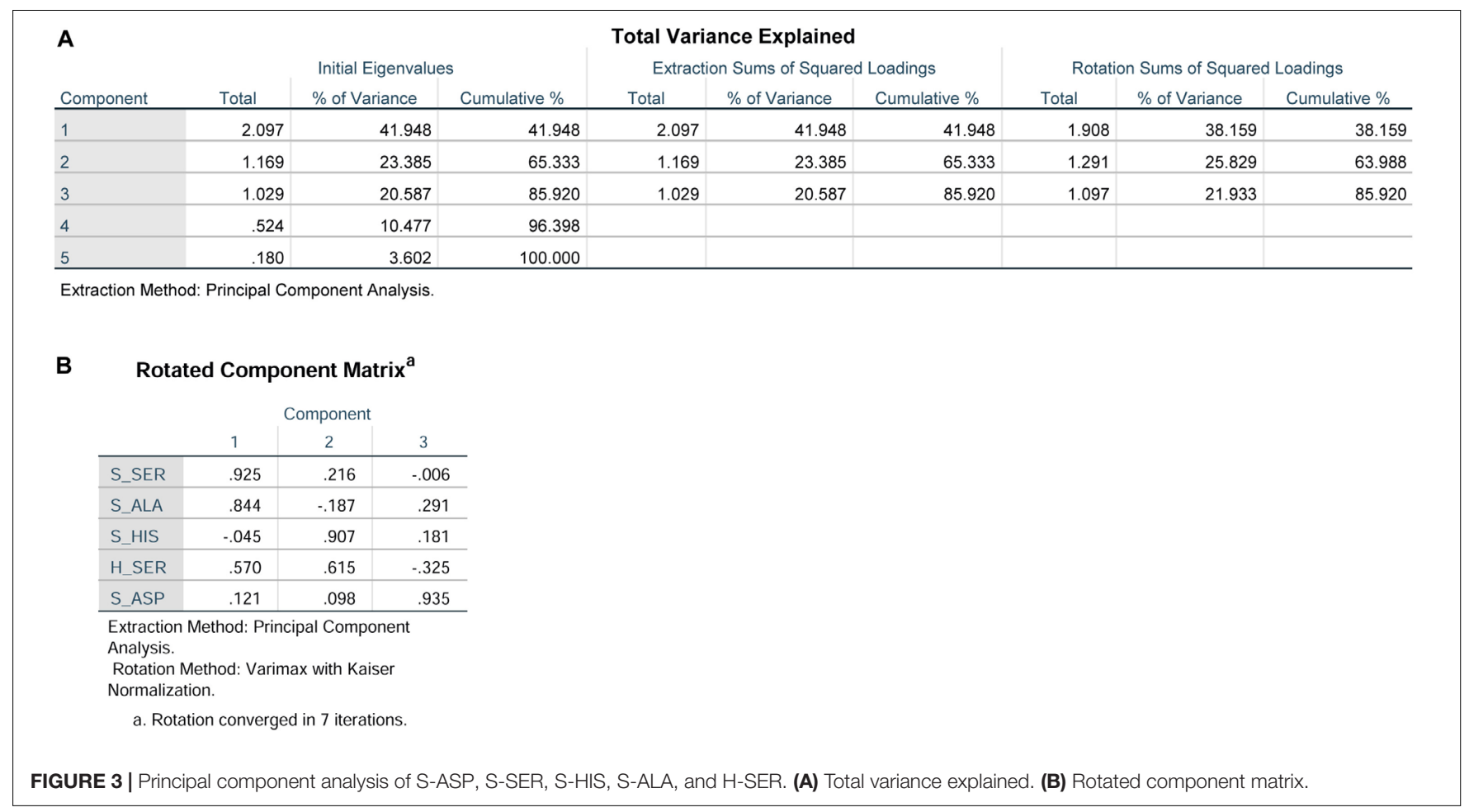

prediction rate is $93.8 \%$, and the accuracy of the crossvalidation is $92.5 \%$.

\section{PCA in Combination With Discriminant Analysis for Amino Acid Date}

Principal component analysis was used to reduce the dimensionality of the dataset and provide an objective amalgamation of the amino acid data. As determined from PCA using included S-ASP, S-SER, S-HIS, S-ALA, and H-SER data, three principle components were extracted (Figure 3A), and the formulas were: F1 $=0.121 S$-ASP $+0.925 S-S E R-$ 0.045S-HIS + 0.884 S-ALA + 0.570 H-SER; F2 = 0.098SASP + 0.216 S-SER + 0.907 S-HIS-0.187 S-ALA + 0.615 H-SER; $\mathrm{F} 3=0.935$ S-ASP-0.006 S-SER + 0.181 S-HIS + 0.291 S-ALA$0.325 \mathrm{H}-\mathrm{SER}$ (Figure 3B).

Further discriminant analysis using the three principle components showed that the prediction rate for the blank control group, non-pregnant group, and pregnant group were 100, 91.3, and 90.4\%, respectively (Figure 4). In addition, the overall prediction rate in this prediction model is $95.2 \%$, and the accuracy of the cross validation is $93.8 \%$ (Figure 4 ).

\section{DISCUSSION}

In the present study, we determined the concentrations of amino acids in D3 embryo culture medium. ASP, SER, GLY, HIS, TAU, ARG, THR, ALA, and PRO were detected in the culture medium. There were significant differences between the pregnant group and non-pregnant group in H-SER, S-ASP, S-SER, S-HIS, and S-ALA. The discrimination analysis according to the peak height and peak area of amino acids revealed that the prediction rate of the pregnant group, non-pregnant group, and blank control group are 82.7, 95.7, and 100\%. In addition, by using the principal component analysis, we found that the prediction rate in these three groups were 90.4, 91.3, and 100\%. Our data may suggest the discrimination model derived from data (amino acid concentrations) for PCA and discriminant analysis has high accuracy in predicting the relationship between amino acid fingerprint and embryo implantation potential.

With the continuous development and improvement in ART, the evaluation of embryonic development potential has been given more and more attention. From a morphological point of view, low-quality embryos have serious morphological abnormalities such as cytoplasmic coarse particles, intracytoplasmic inclusion bodies, or vacuoles. The morphological scores of the pronuclear stage of the embryo mainly include the size, number, and location of the pronucleus and the morphology of cytoplasm (Fragouli et al., 2014). The morphological scores of the cleavage stage mainly include the number and symmetry of the blastomeres, the extent of the fragments, and whether there are vacuoles (Fragouli et al., 2014). Studies have shown that the degree of fragmentation in the embryo on D3 is closely related to the successful rate of embryo implantation (Scott and Smith, 1998). Embryologists have observed that when the fragment rate in the embryo under the microscope is $<10 \%$, its influence can be ignored Alpha Scientists in Reproductive Medicine and ESHRE Special Interest Group of Embryology (2011). However, high fragmentation rates can severely reduce the successful rates of embryo implantation. At the same time, when the fragmentation rate of the embryo is increased from 0 to $35 \%$, the chromosome translocation rate 


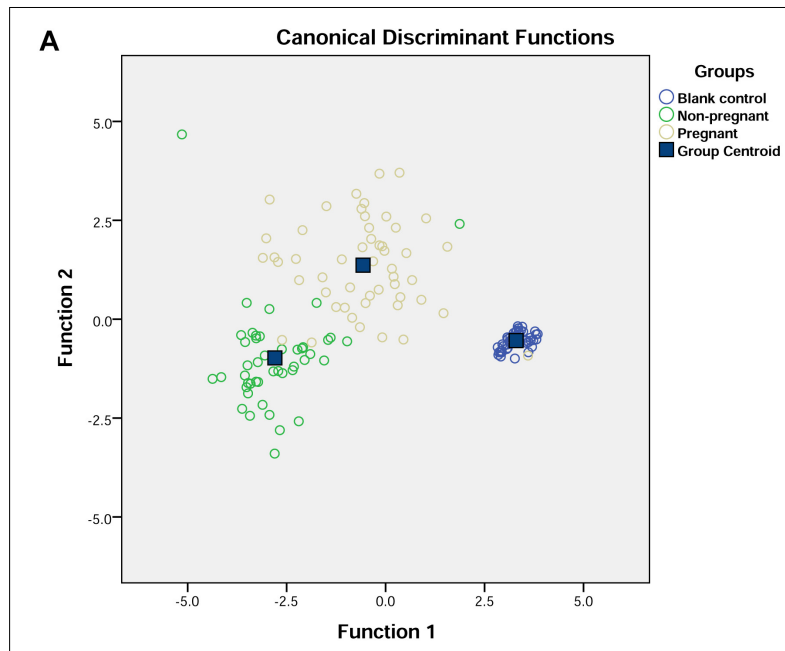

B

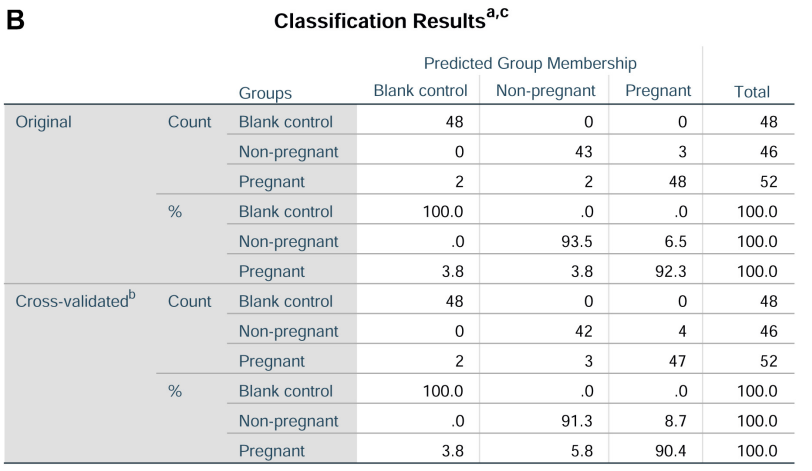

a. $95.2 \%$ of original grouped cases correctly classified.

b. Cross validation is done only for those cases in the analysis. In cross validation, each case is classified by the functions derived from all cases other than that case.

c. $93.8 \%$ of cross-validated grouped cases correctly classified.

FIGURE 4 | Discriminant analysis of the three components from principal component analysis. (A) Canonical discriminant functions. (B) Classification results.

can be increased by $10-30 \%$ Alpha Scientists in Reproductive Medicine and ESHRE Special Interest Group of Embryology (2011). However, the generation and migration of debris is dynamic, so the degree of fragmentation of embryos has not been used as an independent indicator to evaluate the potential of embryo implantation (Athayde Wirka et al., 2014). As women older than 35 years showed decreased function of their ovaries with an increased aneuploidy rate of eggs American College of Obstetricians and Gynecologists Committee on Gynecologic Practice and Practice Committee (2014), these will lead to unreliable morphological evaluation of the embryo. As such, we only included the patients with an age less than 35 for the amino acid profile analysis in the embryo culture medium.

Metabolomics is a new discipline established in the 1990s and is another important component of systems biology following genomics, transcriptomics, and proteomics (Fiehn, 2002; Garlow, 2002; Heijne et al., 2003; Gilchrist et al., 2006; Kern et al., 2007). Amino acid metabolism plays a very important role in early embryonic growth and development (Wu, 2010).
The synthesis of proteins required for embryonic growth and development is closely related to amino acid metabolism. Among them, glutamic acid is formed by amino acid transferase, and further decomposes to produce $\alpha$-ketoglutaric acid, which forms adenosine triphosphate under the action of a citric acid cycle. In addition, glutamate provides a secondary source of carbon and nitrogen for the re-synthesis of pyrimidines and hydrazines, and acts as a reducing agent to protect cells from oxidative stress. Arginine forms nitric oxide through the catalytic action of nitric oxide synthase and is involved in the signal transduction pathway of embryos, which is an essential metabolic pathway for embryonic growth and development (Wu, 2010). Recently, metabolomics have also been applied in ART research. Boyama et al. (2016) tested the relationship between the concentration of homocysteine (Hcy) in the culture medium and the pregnancy outcome, indicating that the concentration of Hcy is inversely related to the pregnancy outcome (Boyama et al., 2016). Montsko et al. (2015) used a high performance liquid chromatographymass spectrometry to analyze the composition of embryo culture medium and found that the amount of conjugated globin$\alpha 1$ fragment was correlated with the outcome of pregnancy during IVF, suggesting that detection and quantitation of the $\alpha-1$ haptoglobin fragment of the culture medium proved to be a useful additional method for identifying non-viable embryos. Lydie et al. studied metabolic profiling of spent embryo culture media using high-resolution nuclear magnetic resonance, and their data showed that embryos with poor development potential consumed more amino acids including proline, threonine, lysine, methionine, tyrosine, and phenylalanine (Nadal-Desbarats et al., 2013). Seli et al. (2008) showed that glutamate concentrations determined by1H NMR were significantly higher in a spent culture media of embryos that resulted in pregnancy and delivery compared to those that failed to implant, and proton NMR spectroscopy predicted the viability of individual embryos with a sensitivity of $88.2 \%$ and a specificity of $88.2 \%$. Brison et al. (2004) used HPLC to detect the concentration of amino acids in an embryo culture medium at $24 \mathrm{~h}$, and found that the turnover of three amino acids, Asn, Gly, and Leu, was significantly correlated with a clinical pregnancy and live birth. These correlations were independent of known predictors, such as female age, basal levels of FSH, embryo cell number, and embryo morphological grade (Brison et al., 2004). Subsequently, Stokes et al. (2007) used the HPLC to analyze the post-thaw amino acid metabolism of human embryos from day two to day three of development and showed a significant difference in the utilization of glutamine, alanine, glycine, glutamate, arginine, and lysine between the thawed embryos which developed to the blastocyst stage and those which arrested prior to blastocyst formation. In addition, this method predicts with $87 \%$ accuracy which frozen-thawed embryo would develop to the blastocyst stage (Stokes et al., 2007). In our experiments, we showed that SER, ASP, HIS, and ALA in the culture medium showed a significant difference between the pregnant group and non-pregnant group. Based on the above evidence, the changes of amino acid profiles were varied among different studies, suggesting the dynamic changes of amino acid metabolism during embryo development. In our study, PCA was used to reduce the dimensionality of the dataset and provide 
an objective amalgamation of the amino acid data. The results showed that the prediction rate in these three groups were 90.4, 91.3 , and $100 \%$, suggesting good predictive accuracy in our study. However, whether changes in the concentrations of these amino acids will lead to changes in the expression of proteins that may be related to embryo development is unknown in the present study. Future studies may be performed to determine the possible mechanistic correlation between amino acids profiles and embryo development potential.

However, in our study, several limitations should be considered. First of all, the culture medium was only collected at day 3 of embryo culture. Whether collection of culture medium at another time point could also generate similar results may require further examination. Secondly, the number of clinical samples were limited, and in the future an increased number of clinical samples as determined by the power calculation should be considered to confirm the current findings. Thirdly, we did not examine whether the metabolic profiles related to the quality of embryos may be determined to further elucidate the predictive role of amino acids profiling in pregnancy outcome in IVF. In the study, we used both the peak height and surface area for the data analysis, while the peak height can be varied among different tests. In order to achieve more reliable prediction, we should optimize these parameters in more clinical samples.

\section{CONCLUSION}

In conclusion, our results showed the significant differences in SER, ASP, HIS, and ALA concentrations in the spent culture media between embryos that resulted in pregnancy and embryos that failed to implant. Furthermore, the discrimination model which derived from the data applied for principal component analysis and discriminant analysis using amino acid data showed a good predictive accuracy for the pregnancy outcome. Although the current amino acid metabolism profile cannot be fully used

\section{REFERENCES}

Alpha Scientists in Reproductive Medicin and ESHRE Special Interest Group of Embryology (2011). Istanbul consensus workshop on embryo assessment: proceedings of an expert meeting. Reprod. Biomed. 22, 632-646. doi: 10.1016/j. rbmo.2011.02.001

American College of Obstetricians and Gynecologists Committee on Gynecologic Practice and Practice Committee (2014). Female age-related fertility decline. Committee Opinion No. 589. Fertil. Steril. 101, 633-634. doi: 10.1016/j. fertnstert.2013.12.032

Armstrong, S., Bhide, P., Jordan, V., Pacey, A., and Farquhar, C. (2018). Timelapse systems for embryo incubation and assessment in assisted reproduction. Cochrane Database Syst. Rev. 5:Cd011320. doi: 10.1002/14651858.CD011320. pub3

Athayde Wirka, K., Chen, A. A., Conaghan, J., Ivani, K., Gvakharia, M., Behr, B., et al. (2014). Atypical embryo phenotypes identified by time-lapse microscopy: high prevalence and association with embryo development. Fertil. Steril. 101, 1637-1648.e1-e5. doi: 10.1016/j.fertnstert.2014.02.050

Borowiecka, M., Wojsiat, J., Polac, I., Radwan, M., Radwan, P., and Zbikowska, H. M. (2012). Oxidative stress markers in follicular fluid of women undergoing in vitro fertilization and embryo transfer. Syst. Biol. Reprod. Med. 58, 301-305. doi: 10.3109/19396368.2012.701367

Boyama, B. A., Cepni, I., Imamoglu, M., Oncul, M., Tuten, A., Yuksel, M. A., et al. (2016). Homocysteine in embryo culture media as a predictor of pregnancy as an independent reference, it can be used in conjunction with morphological assessment methods to increase accuracy in predicting embryo implantation potential.

\section{DATA AVAILABILITY STATEMENT}

All the data in the study is available upon request from the corresponding author.

\section{ETHICS STATEMENT}

This study was approved by the Ethics Committee of Affiliated Hospital of Guilin Medical University. The patients/participants provided their written informed consent to participate in this study.

\section{AUTHOR CONTRIBUTIONS}

$\mathrm{XL}$ and SZ designed the whole study. $\mathrm{PH}$ and $\mathrm{YZ}$ performed all the experiments. JL, PH, YZ, and CL performed the data analysis. JY and LQ performed the statistical analysis. XL and SZ wrote the manuscript. All authors approved the manuscript for submission.

\section{FUNDING}

This study was supported by the Key Project of Guangxi Natural Science Foundation (No. 2018GXNSFDA050003), Guangxi University Young and Middle-aged Teachers Basic Ability Promotion Project (No. 2017KY0477), Guangxi Science Foundation (No. 2017GXNSFBA198189), and National Natural Science Foundation of China (Nos. 81960274, 81860733, and 81860285).

outcome in assisted reproductive technology. Gynecol. Endocrinol. 32, 193-195. doi: 10.3109/09513590.2015.1102877

Brison, D. R., Houghton, F. D., Falconer, D., Roberts, S. A., Hawkhead, J., Humpherson, P. G., et al. (2004). Identification of viable embryos in IVF by non-invasive measurement of amino acid turnover. Hum. Reprod. 19, 23192324. doi: 10.1093/humrep/deh409

De Rose, M. B., Piccolomini, M. M., Soares Belo, A. S., Borges, E. Jr., and Filho, F. F. (2018). Proteomics in human reproduction. Protein Pept. Lett. 25, 420-423. doi: 10.2174/0929866525666180412164602

Egea, R. R., Puchalt, N. G., Escriva, M. M., and Varghese, A. C. (2014). OMICS: current and future perspectives in reproductive medicine and technology. J. Hum. Reprod. Sci. 7, 73-92. doi: 10.4103/0974-1208.138857

Fiehn, O. (2002). Metabolomics-the link between genotypes and phenotypes. Plant Mol. Biol. 48, 155-171. doi: 10.1007/978-94-010-0448-0_11

Fragouli, E., Alfarawati, S., Spath, K., and Wells, D. (2014). Morphological and cytogenetic assessment of cleavage and blastocyst stage embryos. Mol. Hum. Reprod. 20, 117-126. doi: 10.1093/molehr/gat073

Garlow, S. J. (2002). And now, transcriptomics. Neuron 34, 327-328. doi: 10.1016/ s0896-6273(02)00680-3

Gilchrist, A., Au, C. E., Hiding, J., Bell, A. W., Fernandez-Rodriguez, J., Lesimple, S., et al. (2006). Quantitative proteomics analysis of the secretory pathway. Cell $127,1265-1281$.

Heijne, W. H., Stierum, R. H., Slijper, M., Van Bladeren, P. J., and Van Ommen, B. (2003). Toxicogenomics of bromobenzene hepatotoxicity: a combined 
transcriptomics and proteomics approach. Biochem. Pharmacol. 65, 857-875. doi: 10.1016/s0006-2952(02)01613-1

Kern, A., Tilley, E., Hunter, I. S., Legisa, M., and Glieder, A. (2007). Engineering primary metabolic pathways of industrial micro-organisms. J. Biotechnol. 129, 6-29. doi: 10.1016/j.jbiotec.2006.11.021

Li, X. X., Cao, P. H., Han, W. X., Xu, Y. K., Wu, H., Yu, X. L., et al. (2018). Non-invasive metabolomic profiling of culture media of ICSI- and IVF-derived early developmental cattle embryos via Raman spectroscopy. Anim. Reprod. Sci. 196, 99-110. doi: 10.1016/j.anireprosci.2018. 07.001

Liberman, R. F., Getz, K. D., Heinke, D., Luke, B., Stern, J. E., Declercq, E. R., et al. (2017). Assisted reproductive technology and birth defects: effects of subfertility and multiple births. Birth Defects Res. 109, 1144-1153. doi: 10.1002/bdr2. 1055

Montsko, G., Zrinyi, Z., Janaky, T., Szabo, Z., Varnagy, A., Kovacs, G. L., et al. (2015). Noninvasive embryo viability assessment by quantitation of human haptoglobin alpha- 1 fragment in the in vitro fertilization culture medium: an additional tool to increase success rate. Fertil. Steril. 103, 687-693. doi: 10.1016/ j.fertnstert.2014.11.031

Nadal-Desbarats, L., Veau, S., Blasco, H., Emond, P., Royere, D., Andres, C. R., et al. (2013). Is NMR metabolic profiling of spent embryo culture media useful to assist in vitro human embryo selection? Magma 26, 193-202. doi: 10.1007/ s10334-012-0331-x

Nel-Themaat, L., and Nagy, Z. P. (2011). A review of the promises and pitfalls of oocyte and embryo metabolomics. Placenta 32(Suppl. 3), S257-S263. doi: 10.1016/j.placenta.2011.05.011

Rodgaard, T., Heegaard, P. M., and Callesen, H. (2015). Non-invasive assessment of in-vitro embryo quality to improve transfer success. Reprod. Biomed. 31, 585-592. doi: 10.1016/j.rbmo.2015.08.003

Scoccia, B. (2015). What is new in assisted reproduction and multiple pregnancy reduction?: best articles from the past year. Obstet. Gynecol. 126, 446-447. doi: 10.1097/aog.0000000000000979
Scott, L. A., and Smith, S. (1998). The successful use of pronuclear embryo transfers the day following oocyte retrieval. Hum. Reprod. 13, 1003-1013. doi: 10.1093/ humrep/13.4.1003

Seli, E., Botros, L., Sakkas, D., and Burns, D. H. (2008). Noninvasive metabolomic profiling of embryo culture media using proton nuclear magnetic resonance correlates with reproductive potential of embryos in women undergoing in vitro fertilization. Fertil. Steril. 90, 2183-2189. doi: 10.1016/j.fertnstert.2008.07.1739

Stokes, P. J., Hawkhead, J. A., Fawthrop, R. K., Picton, H. M., Sharma, V., Leese, H. J., et al. (2007). Metabolism of human embryos following cryopreservation: implications for the safety and selection of embryos for transfer in clinical IVF. Hum. Reprod. 22, 829-835. doi: 10.1093/humrep/del447

Sturmey, R. G., Brison, D. R., and Leese, H. J. (2008). Symposium: innovative techniques in human embryo viability assessment. Assessing embryo viability by measurement of amino acid turnover. Reprod. Biomed. 17, 486-496. doi: 10.1016/s1472-6483(10)60234-9

Wu, G. (2010). Functional amino acids in growth, reproduction, and health. $A d v$. Nutr. 1, 31-37. doi: 10.3945/an.110.1008

Zhou, Z., Zheng, D., Wu, H., Li, R., Xu, S., Kang, Y., et al. (2018). Epidemiology of infertility in China: a population-based study. BJOG 125, 432-441. doi: 10.1111/1471-0528.14966

Conflict of Interest: The authors declare that the research was conducted in the absence of any commercial or financial relationships that could be construed as a potential conflict of interest.

Copyright (c) $2020 \mathrm{Huo}$, Zhu, Liang, Yao, Le, Qin, Lei and Zhang. This is an openaccess article distributed under the terms of the Creative Commons Attribution License (CC BY). The use, distribution or reproduction in other forums is permitted, provided the original author(s) and the copyright owner(s) are credited and that the original publication in this journal is cited, in accordance with accepted academic practice. No use, distribution or reproduction is permitted which does not comply with these terms. 